Revue Française de Civilisation Britannique

\title{
Le Programme du parti travailliste aux élections législatives de 2019 : la plus longue liste de Noël de l'histoire?
}

The 2019 Labour Party General Election Manifesto: the Longest Christmas Wish

List in History?

\section{Toufik Abdou}

\section{OpenEdition}

Journals

Édition électronique

URL : http://journals.openedition.org/rfcb/5827

DOI : $10.4000 /$ rfcb.5827

ISSN : 2429-4373

Éditeur

CRECIB - Centre de recherche et d'études en civilisation britannique

Référence électronique

Toufik Abdou, «Le Programme du parti travailliste aux élections législatives de 2019 : la plus longue liste de Noël de l'histoire? », Revue Française de Civilisation Britannique [En ligne], XXV-3 | 2020, mis en ligne le 10 septembre 2020, consulté le 10 septembre 2020. URL : http://journals.openedition.org/ rfcb/5827 ; DOI : https://doi.org/10.4000/rfcb.5827

Ce document a été généré automatiquement le 10 septembre 2020.

\section{c) (i) $\Theta$}

Revue française de civilisation britannique est mis à disposition selon les termes de la licence Creative Commons Attribution - Pas d'Utilisation Commerciale - Pas de Modification 4.0 International. 


\title{
Le Programme du parti travailliste aux élections législatives de 2019 : la plus longue liste de Noël de l'histoire?
}

\author{
The 2019 Labour Party General Election Manifesto: the Longest Christmas Wish \\ List in History?
}

Toufik Abdou

\section{Introduction}

1 Le parti travailliste a connu l'un des plus mauvais scores de son histoire aux élections législatives britanniques de décembre 2019. Quelques jours après sa pire défaite depuis 1935, le chef de l'opposition Jeremy Corbyn a présenté ses excuses à ses électeurs dans les colonnes de l'observer:

«Même si nous avons subi de lourdes pertes en termes de sièges jeudi dernier, je pense que le programme de 2019 et le mouvement qui l'a accompagné auront une importance historique, ce fut une réelle tentative pour construire une force suffisamment puissante pour transformer la société, dans l'intérêt du plus grand nombre, et non pas d'une minorité. $»^{1}$.

Dans ce mea culpa, Jeremy Corbyn a mis la défaite sur le compte de l'extrême polarisation de la vie politique britannique autour de la question du Brexit tout en réfutant les attaques contre son programme de 2019. Corbyn reste en effet persuadé d'avoir "gagné le débat " ${ }^{2}$, d'avoir mis fin au dogme de l'austérité et d'avoir réussi à ancrer le parti travailliste fermement à gauche. Nous essaierons de définir ici les grandes orientations idéologiques du parti travailliste et d'envisager le Corbynisme à travers le prisme du programme 2019. 


\section{Le programme travailliste le plus à gauche depuis 1983}

3 Les programmes électoraux sont des bréviaires qui nous montrent ce que ferait un parti s'il accédait au pouvoir. Ce sont également des guides utiles et précis qui reflètent l'évolution du parti. Tout comme les rapports du congrès annuel du parti travailliste, les programmes sont une source primaire précieuse. Ils offrent une grille de lecture et permettent de retracer la trajectoire idéologique d'un parti qui a offert depuis 2010 quatre orientations politiques qui se sont soldées par quatre défaites successives. " L'absence de cadre idéologique clairement défini fait du parti travailliste une institution tout particulièrement britannique $»^{3}$. Très peu de dirigeants travaillistes ont en effet "ressenti le besoin de justifier la pratique gouvernementale par le biais d'un théorie générale $»^{4}$. A cet égard, les programmes électoraux fournissent un matériau utile pour qui souhaite étudier l'évolution idéologique du parti travailliste, notamment lorsqu'il est dans l'opposition. Le pragmatisme et l'ouverture du parti capable d'intégrer des opinions diverses, ainsi que l'indique la traditionnelle description du parti comme une «broad church » explique la pluralité et l'étendue des propositions politiques exposées dans les programmes du parti travailliste. Définir les contours de la pensée politique du parti travailliste est une tâche ardue, le travaillisme étant une idéologie protéiforme qui relève d'" une approche pragmatique où la tradition et l'expérience pèsent plus que les abstractions intellectuelles $»^{5}$.

4 Les partis politiques britanniques sont programmatiques. Toute élection législative confère à un parti un mandat pour diriger le pays. Selon la Convention de Salisbury, une victoire électorale donne ainsi à un parti l'autorité et la légitimité pour mettre en œuvre les promesses électorales inscrites dans son programme. Les batailles électorales se disputent en effet autour des programmes électoraux et, s'ils sont élus, les dirigeants des partis politiques promettent de les mettre en œuvre. L'importance du programme est intrinsèquement liée au système bipartisan britannique. Samuel Edward Finer fait ainsi remarquer que les fortes majorités parlementaires tendraient à renforcer les antagonismes, les oppositions partisanes et encourageraient les partis à proposer un contre-projet $^{6}$. Les programmes électoraux permettent également aux chercheurs d'analyser l'évolution idéologique d'un parti. C'est ce que propose de faire le Manifesto Project Database (MPD), la base de données complète des programmes politiques compilée par le projet MARPOR, l'acronyme du Manifesto Research on Political Representation ${ }^{7}$. Ce projet utilise les méthodes quantitatives afin d'analyser les traits saillants des programmes des partis de plus de 50 pays démocratiques depuis 1950. Les codeurs divisent le texte en unités de sens et le classent dans un logiciel afin d'en évaluer le positionnement idéologique. Le programme de 2019 est plus radical que celui de 2017 qui était déjà le plus à gauche depuis 1992. Le graphique ci-dessous montre que le tournant à gauche a commencé en 2015 sous l'ère Ed Miliband. A ce jour, le programme de 2019 n'a toujours pas été codé mais il est fort probable que celui-ci se rapproche du programme du 1983. Le texte de Michael Foot très ancré à gauche est resté dans les annales comme « la plus longue lettre de suicide de l'histoire $»^{8}$. 


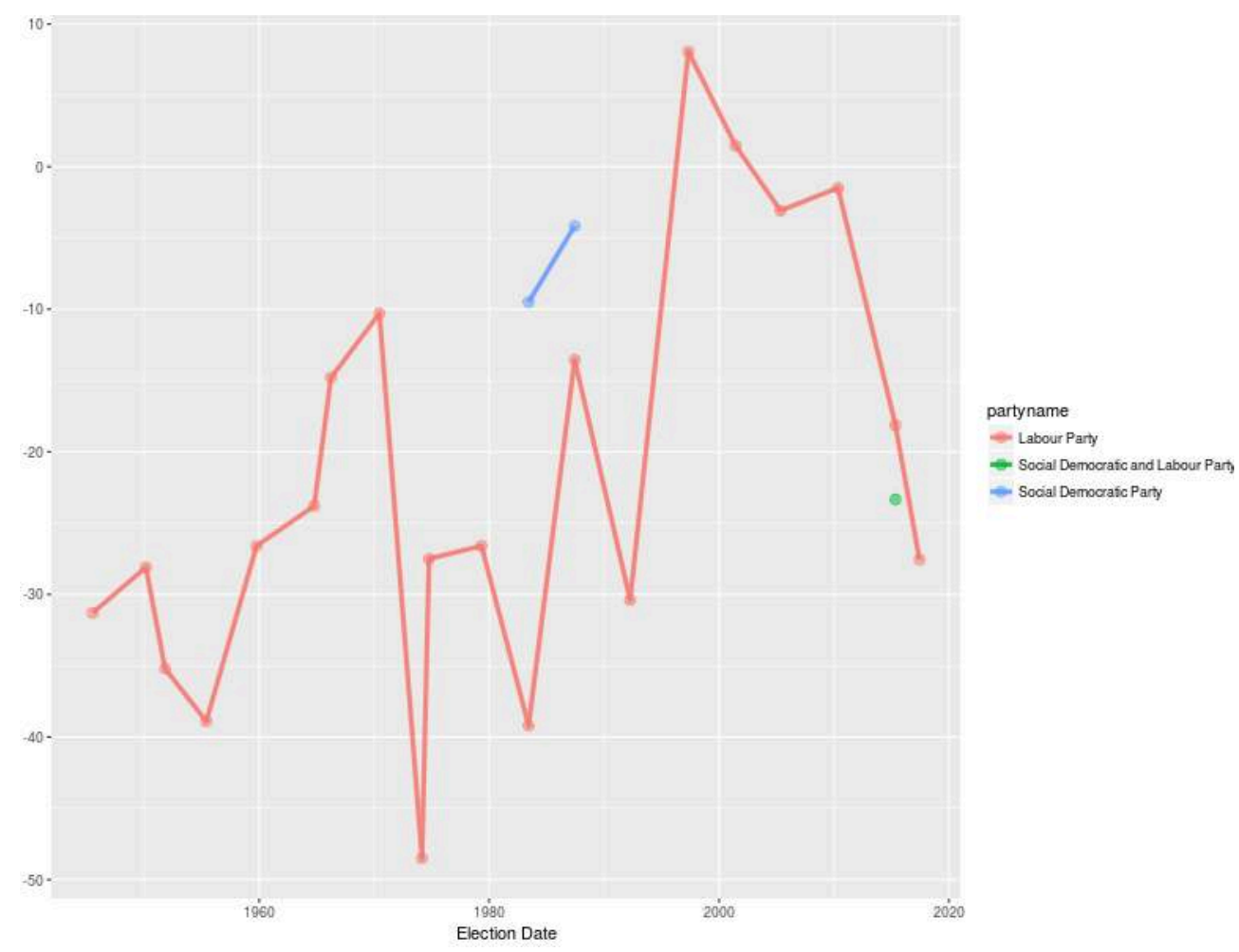

Figure 1 : Evolution idéologique du parti travailliste entre 1983 et $2017^{9}$

\section{Le programme économique 2019 : Jeremy Corbyn « verdit » son discours}

Avec ses 105 pages, le programme du parti travailliste version 2019 se caractérise par sa prolixité. En 2017, le premier programme de Jeremy Corbyn se déclinait déjà en douze chapitres sur un total 126 pages. Le découpage innovant de 2019 illustre les grands principes idéologiques du Corbynisme : La Révolution Industrielle Verte, Reconstruire Nos Services Publics, Combattre la Pauvreté et les Inégalités, le Dernier Mot sur le Brexit, un Nouvel Internationalisme.

Lors de la présentation du programme à la presse, Jeremy Corbyn a résumé ses mesures économiques comme un "blitz d'investissements". Les travaillistes ont en effet promis de créer un Fonds de Transformation Nationale de 400 milliards de livres afin de reconstruire les infrastructures britanniques. 250 milliards furent réservés au Fonds de Transformation Verte dédié à la conversion environnementale des logements et de l'appareil industriel. La grande différence entre le programme 2017 et celui de 2019 réside dans l'intégration de la question environnementale dans le discours politique de Jeremy Corbyn. La révolution industrielle verte que Jeremy Corbyn appelait de ses vœux s'inspirait du «Green New Deal » défendu par la sénatrice américaine Alexandria Ocasio-Cortez. Il s'agissait d'éliminer les émissions de gaz à effet de serre et de moderniser les infrastructures tout en réduisant les fractures sociales et géographiques. En outre, les travaillistes avaient prévu de créer une Banque Nationale d'Investissement, épaulée par un réseau de Banques Régionales de Développement, afin d'accorder des prêts d'une valeur de 250 milliards de livres sur 10 ans visant à favoriser l'innovation et la création d'entreprises. L'objectif était de compenser l'extrême 
centralisation au Royaume-Uni. Une étude publiée en septembre 2019 par l'Université de Sheffield a en effet démontré que le Royaume-Uni détient le record des inégalités régionales les plus criantes parmi 29 pays de l'OCDE ${ }^{10}$. Le recours au mot «transformation" n'est pas anodin. Avec 23 occurrences dans le programme 2019, le lexème faisait partie de la matrice idéologique de Jeremy Corbyn. On voit ici l'influence de l'économiste hongrois Karl Polanyi et de son ouvrage phare La Grande Transformation ${ }^{11}$. En 1944, Karl Polanyi expliquait les difficultés rencontrées par le capitalisme entre les deux guerres. Elles résultaient de la tendance à instaurer un marché autorégulateur depuis le $19^{\mathrm{ème}}$ siècle. Polanyi attendait donc une ré-articulation de l'économie et de la société à travers la planification sociale ${ }^{12}$. C'est cette pensée polanyienne qui alimente les débats entre les «Lexiters "13 qui rêvent d'un Brexit et d'un Corbynisme total qui s'articuleraient autour de la notion de souveraineté nationale dans les domaines du commerce, de la monnaie et de la politique économique afin de « contrebalancer les forces du néo-libéralisme international $»^{14}$.

8 Corbyn a promis de supprimer le crédit universel, le système d'aide sociale extrêmement controversé. Le programme 2019 prévoyait également de mettre fin aux coupes budgétaires en investissant par exemple 22 milliards de livres dans la formation de nouveaux médecins et la rénovation des hôpitaux de la NHS. La poste, les chemins de fer, l'eau, l'électricité et les réseaux de bus municipaux devaient revenir dans le domaine public :

Le parti travailliste s'assurera que les municipalités puissent améliorer les services de bus en régulant et en remettant les réseaux de bus dans le domaine public (...) Lorsque les mairies reprendront le contrôle de leurs bus, le parti travailliste instaurera la gratuité pour les moins de 25 ans ${ }^{15}$.

Cette mesure estimée à 1,4 milliard de livres et financée par la vignette automobile ${ }^{16}$, s'inscrivait dans le programme social et écologique de Jeremy Corbyn et aurait permis de venir en aide aux jeunes qui votent désormais majoritairement pour le parti travailliste. La disparition de 3000 lignes de bus ${ }^{17}$ est devenue le symbole des politiques d'austérité mises en œuvre par les conservateurs depuis 2010. C'est en ces termes qu'Andy McDonald, le Ministre des Transports du Cabinet fantôme de Jeremy Corbyn a défendu cette mesure :

Les Conservateurs ont négligé les bus de ville, ainsi que les personnes et les communautés qui en dépendent. Sabrer dans le budget des bus de ville nuit à nos communautés en déconnectant les personnes du monde du travail et des loisirs tout en aggravant la congestion de nos routes et la pollution de l'air. ${ }^{18}$

Lisa Nandy, candidate à la direction du parti en 2020 et chantre du modèle de Preston ${ }^{19}$, de la décentralisation et du travaillisme municipal, a fait du rétablissement du réseau de transports publics l'un de ses chevaux de bataille.

11 Un gouvernement Corbyn aurait étendu la gratuité des services publics à la fibre optique et aurait lancé la construction de 500000 logements sociaux. En revanche, contrairement à ce qui avait été annoncé lors du Congrès du parti travailliste de septembre 2019, les écoles privées n'auraient pas été nationalisées. Le refus d'inclure les mesures-phares mais très radicales du Congrès 2019 telles que l'abolition des écoles privées, la libre circulation des migrants européens après le Brexit et la promesse d'inscrire dans la loi l'objectif de neutralité carbone dès $2030^{20}$ tendrait à confirmer la thèse selon laquelle les dirigeants du parti travailliste utiliseraient leur pouvoir de modération afin d'accroitre leur éligibilitée ${ }^{1}$. 

des impôts des grandes entreprises (passant de $21 \%$ à $26 \%$ ) et des actionnaires. Une taxation plus lourde aurait également touché les contribuables aux revenus supérieurs à 80000 livres (les 5\% les plus riches). Le programme de 2019 prévoyait des augmentations d'impôts de l'ordre de 82,9 milliards de livres par an, en forte augmentation comparativement aux 48,6 milliards du programme 2017. Selon Ben Zaranko, économiste à l'Institute for Fiscal Studies, le projet du parti travailliste aurait porté la dépense publique à un niveau jamais vu depuis les années 1976-197722. gouvernements conservateurs. Le programme économique de Corbyn remettait en question le dogme de l'austérité instauré depuis 2010 en proposant par exemple une augmentation de $5 \%$ du salaire des infirmières, des officiers de police et des enseignants. Richard Murphy, un comptable spécialiste en droit fiscal, est à l'origine de nombreuses idées de la plateforme économique de Corbyn, notamment le rejet du paradigme de l'austérité, la création d'une banque publique d'investissement et la théorie de l'assouplissement quantitatif au profit du peuple désormais surnommée " Corbynomics $»^{23}$. En économie, l'assouplissement quantitatif désigne les politiques monétaires non conventionnelles mobilisées lors de crises exceptionnelles.

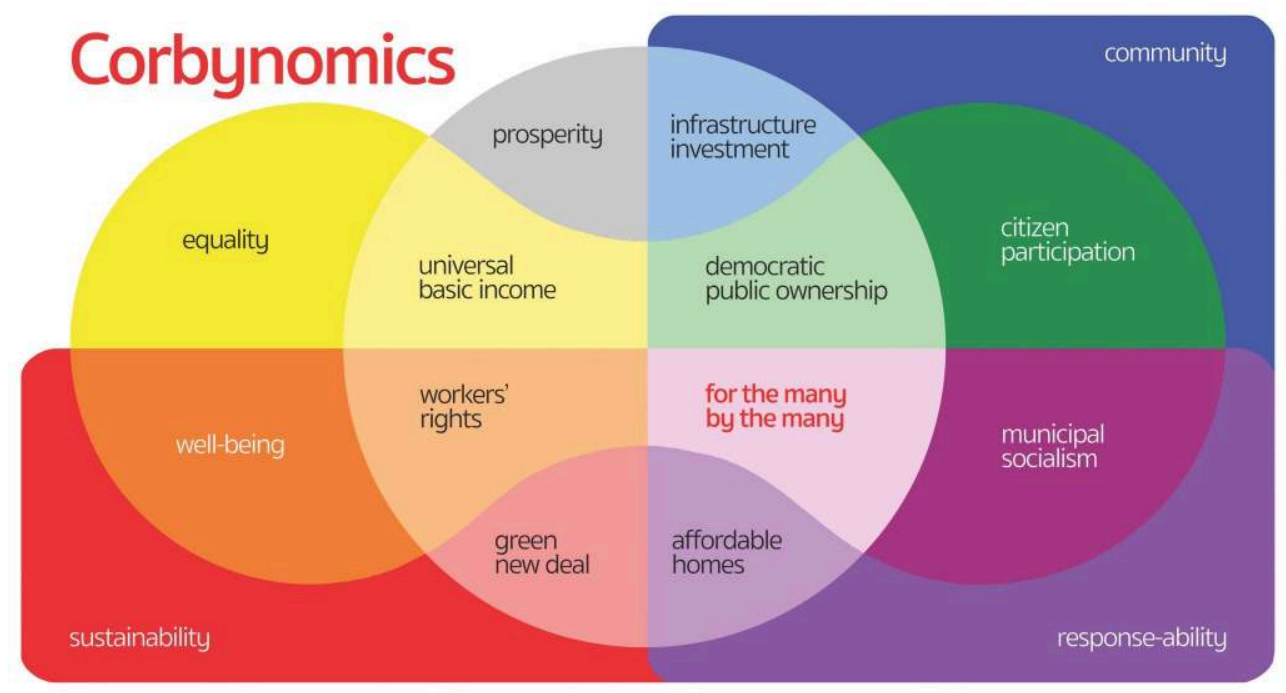

Figure 2 : Les grands principes économiques du Corbynomics ${ }^{24}$

Le document «Alternative Models of Ownership » (AMO) produit en 2017 sous la houlette de Rebecca Long Bailey et John McDonnell définissait les grandes orientations économiques de Jeremy Corbyn. L'AMO soutenait que les "failles structurelles fondamentales ${ }^{25}$ de l'économie britannique étaient dues au manque d'investissements à long terme et à la baisse de la productivité qui «ont nui à la démocratie, ont conduit les régions du pays à l'oubli économique et ont contribué à des niveaux croissants d'inégalités et d'insécurité financière $»^{26}$. C'est par la planification, la promotion des coopératives, la décentralisation et le nationalisme économique que le parti travailliste souhaitait régler ces problèmes structurels. Ces outils économiques devaient également entraîner un partage plus équitable des retombées économiques de la robotisation ${ }^{27}$.

Corbyn s'inspirait du post-capitalisme et de la techno-utopie ${ }^{28}$ théorisés au RoyaumeUni par les journalistes Paul Mason et Aaron Bastani, Nick Srniceck et Alex Williams, auteurs d'Accélérer le futur: Post-travail \& Post-capitalisme ${ }^{29}$ et le think tank Autonomy. 
Cette théorie politique post-capitaliste est une réinterprétation, la version 2.0 de la théorie marxiste du matérialisme historique. Le rôle de l'Etat n'est pas de protéger les emplois obsolètes mais de mettre en place des politiques sociales adéquates pour faire face aux défis de la mondialisation ${ }^{30}$. Ce n'est plus la lutte des classes qui donne une direction à l'histoire mais le changement technologique :

Nous lancerons le plus grand programme d'investissements de l'ère moderne afin de financer les emplois et les industries du futur, afin que personne ne soit freiné et qu'aucune communauté ne soit abandonnée. Ceci est un programme entièrement chiffré dont le but est de moderniser notre économie et transformer notre pays. ${ }^{31}$

17 La classe ouvrière (une seule référence dans le programme 2019) n'a plus de rôle historique. Elle est remplacée par les cyber-militants de Momentum ${ }^{32}$ qui ont réussi à cibler les électeurs indécis grâce au logiciel Promote ${ }^{33}$ en 2017 ou l'armée de jeunes clicktivistes connectés à la toile qui ont poussé "Jezza " $^{34}$ à se présenter aux élections primaires du parti travailliste en 2015. D'ailleurs, la mesure la plus controversée du programme 2019 fut assurément celle de rendre gratuite la fibre optique. Cette proposition qui fut moquée par le PDG de British Telecom comme étant du « communisme à haut débit $»^{35}$ avait pour but d'accroitre la productivité et la compétitivité du Royaume-Uni. Selon Jeremy Corbyn, l'Internet devrait devenir l'« institution nationale du $21^{\text {ème }^{2}}$ siècle » tout comme la NHS fut «l'institution la plus précieuse du $20^{\text {ème }}$ siècle »"

\section{Un programme ancré dans l'histoire du parti}

18 Le thème du coût de la vie et les «bread and butter issues » prenaient une large place dans le programme 2019. Jeremy Corbyn promettait de faciliter l'accès à la propriété, d'augmenter le salaire minimum, d'interdire les contrats zéro heure et de supprimer la très symbolique "bedroom tax ». Il allait plus loin en offrant de supprimer les tarifs d'entrée à l'Université. Deux mouvements de contestation en particulier ont joué un rôle crucial dans l'ascension du vétéran du parlement. D'une part, les manifestations étudiantes de 2010 contre le triplement des frais d'université. L'occupation des locaux du Parti conservateur a déclenché l'un des mouvements les plus importants de l'histoire récente britannique : plus de 100000 jeunes sont descendus dans les rues, et une douzaine de facultés ont été occupées. Cristallisant la colère engendrée par la crise financière de $2008^{37}$, la contestation étudiante a impulsé dans son sillage un mouvement anti-austérité plus global, organisé par des collectifs tels que «Occupy London" et "UK Uncut ». Le deuxième socle de son assise politique fut le mouvement anti-guerre. Corbyn a présidé la coalition «Stop the War", qui a organisé la manifestation de deux millions de personnes contre l'invasion de l'Irak en 2003, la plus grande protestation de masse de l'histoire britannique. Dans la partie du programme consacrée à la politique étrangère, Corbyn mettait ainsi l'accent sur la négociation, la coopération, le droit international et la résolution des conflits ${ }^{38}$.

19 Corbyn ne faisait pas table rase du passé, il se plaçait en effet dans la lignée des leaders travaillistes modernisateurs. Lors de la présentation du programme 2019, John McDonnell, le Chancelier de l'Echiquier du Cabinet fantôme, n'a pas hésité à revendiquer l'héritage du New Labour et notamment ses ambitions réformatrices dans le domaine de l'éducation (le programme 2019 reprend l'initiative Sure Start de Tony Blair) $)^{39}$, l'investissement massif dans les services publics de santé sous le gouvernement Gordon Brown ou le programme anti-austérité d'Ed Miliband en 2015. 
20 " Aujourd'hui, nous dévoilons un programme dont le but est de transformer le $21^{\text {ème }}$ siècle comme Harold Wilson l'a fait en 1964 pour transformer le $20^{\text {ème }}$ siècle » : c'est ce que Jeremy Corbyn avait déclaré lors du lancement de son programme 2017 à Bradford $^{40}$. La promesse de connecter complètement le réseau internet britannique à la fibre (92\%), de prolonger la ligne à grande vitesse HS2 jusqu'à l'Ecosse, de développer les nouvelles technologies de capture du carbone et de construire une économie verte ne sont pas sans rappeler les discours visionnaires d'Harold Wilson sur la révolution scientifique. En 2019, Jeremy Corbyn embrassait la modernité (11 occurrences des mots «moderne » et «moderniser ») et présentait le parti travailliste comme une force du changement (mot répété 47 fois) capable de faire tomber «les barrières " (expression prononcée 4 fois). Dans le programme 2019, le parti conservateur fut en revanche accusé de « freiner » les Britanniques (l'expression « held back » était un des éléments de langage fétiches de l'équipe Corbyn), de dresser des barrières (10 occurrences) qui entravent le progrès. Les Conservateurs (l'expression "Tories", connotée péjorativement, est répétée 25 fois) furent associés à des images qui suggèrent la violence sociale, la division ( « les Conservateurs essaient de nous dresser les uns contre les autres » $\left.{ }^{41}\right)$, ou l'absence d'empathie : «le filet de sécurité sociale britannique a été délibérément supprimé et remplacé par une morale sévère et insensible $\$^{42}$, la "cruauté et le manque de cœur des Conservateurs ont transformé le Ministère du Travail et des Retraites en un symbole de peur $»^{43}$, « les Conservateurs ont gaspillé une décennie en servant les intérêts des grands pollueurs $»^{44}$, leur rhétorique autour « des profiteurs et des tire-au-flanc a attisé la haine à l'égard des personnes en situation de handicap ${ }^{45}$.

21 Corbyn souhaitait pour sa part réparer les maux de la société britannique. On note 60 occurrences du mot «soin» et ses dérivés dans le programme 2019. Sur le modèle du National Health Service (NHS), le National Care Service avait pour but de financer les soins à domicile, notamment des personnes âgées. Jeremy Corbyn reprenait ici une idée contenue dans le programme de Gordon Brown ${ }^{46}$. Le National Education Service (NES) que Corbyn comptait créer aurait fourni une formation gratuite tout au long de la vie. Lors de la présentation de cette nouvelle institution en 2017, Jeremy Corbyn se plaçait en digne héritier de Clement Attlee en proposant de renforcer l'Etat-Providence: «Lorsqu'en 1945, le gouvernement a mis en place la NHS, il a créé une des institutions centrales garantissant l'égalité au $20^{\text {ème }}$ siècle. Le NES fera la même chose pour le $21^{\text {ème }}$ $»^{47}$.

En 2010, Gordon Brown se présentait en expert. En 2015, Ed Miliband projetait l'image d'un homme politique éthique. Le député d'Islington préfère, quant à lui, adopter une rhétorique de l'authenticité. Les leaders authentiques tentent de promouvoir un " climat éthique positif» et sont animés par une "perspective morale internalisée $»^{48}$. Les biographies de Jeremy Corbyn mettent particulièrement l'accent sur l'exemplarité et l'ascétisme d'un leader souvent décrit comme vivant une existence austère : il ne boit pas, ne fume pas, n'a aucun désir de posséder des biens matériels ou d'intérêt pour la culture ; pour lui, la politique est « une passion si dévorante qu'elle ne laisse que peu de place à d'autres intérêts, hobbies ou même des vices »" " Si votre couur bat pour Jeremy Corbyn, vous devriez vous faire transplanter $\aleph^{50}$, avait cru bon de plaisanter Tony Blair, en juillet 2015. Dans le programme de 2019, Jeremy Corbyn construisait l'image d'un leader empathique. Il promettait de rendre leur dignité (mot répété 7 fois) aux travailleurs et de supprimer le système du Crédit Universel afin de le remplacer par « un système 
alternatif qui traite les gens avec dignité et respect $»^{51}$. Le parti travailliste "ne diabolisera jamais les personnes en situation de handicap ou sans emploi ". Corbyn promettait d'apporter son «soutien" (85 mentions) aux plus vulnérables. On note 67 occurrences des mots « protéger » et " protection », 6 du terme « sauvegarder ».

Cette rhétorique de l'authenticité prenait parfois des accents populistes lorsque Jeremy Corbyn proposait de défendre le peuple : «Pour beaucoup de gens, la politique ne fonctionne pas. La bulle de Westminster se situe à des années-lumière de leur vie quotidienne. Le parti travailliste a été fondé pour donner aux classes ouvrières une voix en politique $»^{52}$. L'émergence de Corbyn s'est inscrite dans le cycle de mouvements d'un type nouveau qui ont vu le jour aux Etats-Unis ( Occupy Wall Street»), en Espagne («Podemos») ou en Italie (le mouvement "Cinque Stelle»). Plutôt que de les classer à gauche, le théoricien politique Ernesto Laclau ${ }^{53}$ et la philosophe Chantal Mouffe ${ }^{54}$ préfèrent parler de populisme de gauche, selon une démarche qui polarise le peuple (les $99 \%$ de la population) contre les élites (1\% qui amasse les richesses et les pouvoirs de tout ordre).

L'exigence de justice (mot répété 45 fois) fut au cœur du projet politique de Corbyn. Il promettait de "protéger davantage les lanceurs d'alerte " ${ }^{55}$, de terminer l'enquête sur l'incendie de la tour Grenfell, de rouvrir les dossiers de la bataille d'Orgreave ${ }^{56}$, les procès de Shrewsbury ${ }^{57}$ et des ouvriers du chantier naval du 37 Cammell Laird $^{58}$. Dans ces trois affaires, des grévistes ou des militants syndicalistes furent incarcérés suite à des affrontements avec les forces de l'ordre. Afin de protéger les droits des travailleurs et mettre fin à toute forme d'exploitation dans le monde du travail, Corbyn promettait de renforcer le pouvoir des syndicats (25 occurrences du mot en 2019, 19 en 2017, 1 en 2015, 2 en 2010) car c'est "seulement en déplaçant l'équilibre des pouvoirs du côté des travailleurs que nous réussirons à obtenir des salaires décents, la sécurité et la dignité au travail ${ }^{59}$. Le rétablissement des droits syndicaux fut au cœur du programme social de Jeremy Corbyn, leader très proche des syndicats. Sous la pression de leurs bases, les deux grandes formations syndicales, Unite et Unison, ont apporté un soutien décisif à Corbyn. Une semaine après la remarque de Tony Blair citée plus haut, Dave Ward, le secrétaire général du Communication Workers Union, un syndicat fort de 110000 membres, a également annoncé son soutien à Corbyn : "Il y a un virus au sein du parti travailliste et Jeremy Corbyn est l'antidote. La mainmise des Blairistes et d'individus tels que Peter Mandelson doit cesser une fois pour toute $»^{60}$.

\section{Un manque de crédibilité, de hiérarchisation et de réalisme}

Lors d'une table ronde qui s'est tenue le 20 février 2020 à l'Université King's College de Londres dans le cadre des célébrations des 120 ans du parti travailliste, Tony Blair a attaqué le manque de réalisme du programme 2019 dont le foisonnement et l'ambition démesurée ne peuvent, selon lui, que susciter le scepticisme :

Le problème de ce programme, je l'ai lu, il fait 97 pages, c'est que plus vous le lisiez, moins vous étiez convaincu. C'était un document incroyable parce que sur chaque page, on pouvait deviner que quelqu'un était venu frapper à la porte pour présenter un plan en dix points à un milliard de livres sur tel sujet.

Selon Tony Blair, ces promesses à profusion sont une preuve de faiblesse de la part du chef du parti travailliste qui n'a pas su résister aux pressions des membres de son Cabinet fantôme, des syndicats et des groupes de pression. Un leader de parti, prêt à 
diriger le pays, doit être capable de dire non. Cette incapacité à trancher est, toujours selon Tony Blair, d'autant plus dommageable que le Labour doit, à chaque élection législative, démontrer qu'il peut gouverner ${ }^{61}$. Les informations parues dans la presse et confirmées par Andrew Fisher, l'auteur des «manifestoes» de 2017 et 2019 semblent appuyer cette hypothèse. Les ingérences de Seumas Milne, d'Andrew Murray et de Karie Murphy, respectivement le directeur de la communication, le conseiller et la cheffe de cabinet de Jeremy Corbyn, n'ont fait qu'ajouter à la confusion ${ }^{62}$ et alourdir un processus de décision déjà complexe. Le "manifesto » est en effet le résultat de longues négociations entre le comité exécutif national (NEC) chargé de faire exécuter les motions du Congrès, le cabinet fantôme et les syndicats. La "mouvementisation » du Labour a ajouté une nouvelle strate à ce mille-feuille décisionnel. Fort de 580000 membres, le plus grand parti européen est non seulement saisi par les logiques militantes de sa base mais subit également les pressions de "Momentum», une organisation créée pour soutenir Jeremy Corbyn et influencer la ligne politique du Labour ${ }^{63}$. Lors du festival d'éducation politique "The World Transformed» organisé en marge du Congrès annuel du parti qui s'est tenu à Brighton en septembre 2019, " Momentum" a d'ailleurs lancé un "Manifesto pour le mouvement", résultat de discussions entre les adhérents, le Professeur Mike Neary, l'activiste Dr Faiza Shaheen, la sociologue Hilary Wainwright ou des ténors du parti tels que John McDonnell et Ed Miliband. C'est à l'issue de débats, appelés laboratoires politiques, autour des questions de l'environnement, de l'éducation, de la santé, des transports et de la justice ${ }^{64}$, que " Momentum » a édité un programme alternatif parfois plus radical que celui publié à l'occasion des élections législatives de 2019. Ce "people-powered manifesto", ce programme écrit par les citoyens n'est que l'aboutissement des diverses formes de démocratie participative et délibérative promues par Jeremy Corbyn pendant son mandat même si cette volonté d'introduire davantage d'horizontalité dans le processus de décision au sein du parti travailliste n'a pas toujours été suivie d'effet (on pense notamment à l'échec de la «Democracy Review »). « Momentum applique les principes du "Big Organising " ${ }^{65}$ théorisés par Becky Bond ${ }^{66}$. L'ancienne conseillère de Bernie Sanders, présente au Congrès en septembre 2019, proposait par exemple aux adhérents de jouer un rôle actif dans le parti.

27 La multiplicité des acteurs impliqués dans l'élaboration du projet politique 2019 peut expliquer le brouillage du message travailliste lors de l'élection législative. Le choix du slogan «It's Time for Real Change » n'a pas su susciter l'enthousiasme parmi les militants ou les électeurs et n'a pas pu marquer les esprits face à la redoutable efficacité du triptyque du parti conservateur «Get Brexit Done». Il faut dire que ce slogan presque éculé fait partie des poncifs de la communication politique. Le libéral Justin Trudeau a par exemple utilisé le slogan «Real Change » en 2015, au Canada. La formule "For The Many Not The Few » est toujours présente sur la couverture mais en petits caractères contrairement à 2017 où ce mantra tiré d'un poème de Percy Bysshe Shelley et répété à l'envi pendant tous les discours de campagne, était devenu la signature du parti travailliste. Le succès de la formule en 2017 s'expliquait par son caractère inclusif. Selon Naomi Klein ${ }^{67}$, l'optimisme de ce message inclusif fut à la fois audacieux et porteur d'espoir face aux politiques de la peur des leaders populistes ou le «Project Fear " de la campagne anti-Brexit. Les cadres du parti ont fini par reconnaitre après l'élection législative 2019 qu'ils n'avaient pas su créer un « récit » qui aurait pu donner du sens à leur programme. 

Final Say on Brexit". Le refus de consacrer plus de trois pages et demi à la question du Brexit montre bien la volonté du parti travailliste de déplacer le débat vers les questions socio-économiques. Le nouvel accord «raisonnable " que promettait Corbyn aurait garanti les droits des consommateurs, des migrants européens, des agriculteurs et de l'industrie piscicole restait toutefois flou et peu réaliste même si les promesses dans ce domaine était beaucoup moins ambitieuse qu'en 2017: "Un gouvernement travailliste travaillera de façon constructive avec l'UE sur les questions vitales d'intérêt commun et pour le bénéfice commun du Royaume-Uni et de l'UE. Mais nous quitterons l'UE »68.

\section{Conclusion} succès de la campagne du parti travailliste. Malgré un agenda construit autour de mesures populaires et un verdissement du discours qui correspond aux préoccupations actuelles, le «manifesto » 2019 n'a pas su créer d'élan populaire. « Nous sommes allés trop loin»: c'est la leçon que semble avoir tiré les députés travaillistes, beaucoup plus modérés que la base militante. Le député Stephen Kinnock a attribué la défaite à trois facteurs : un chef impopulaire perçu comme étant faible et peu compétent, la décision de soutenir un second referendum et un programme politique qu'il compare volontiers à "une liste de vœux de cadeaux de Noël ${ }^{69}$. La profusion des mesures proposées, la stratégie de campagne consistant à égrener une mesure par jour sans les hiérarchiser et l'absence d'un message clair, notamment sur la question européenne, ont entamé la crédibilité du parti et lui ont coûté de nombreux sièges. Paradoxalement, le slogan de 2019 «It's Time for Real Change » était vague et déchargé de toute conflictualité alors que le projet économique avait gagné en précision. En effet, les mesures proposées pour faire face au changement climatique ou aux inégalités sociales et géographiques offraient un contre-projet après dix ans de pouvoir conservateur. Le slogan de 2017, " For The Many, Not The Few » avait eu le mérite d'expliciter que pour servir les intérêts de la majorité sociale, les intérêts des citoyens les plus riches et plus puissants seraient forcément lésés ${ }^{70}$. Paradoxalement également, les mesures telles que la nationalisation des entreprises de l'énergie, des trains et de la poste, la suppression des frais de scolarité à l'Université ou l'augmentation des impôts des contribuables gagnant plus de 80000 livres étaient populaires ${ }^{71}$ mais le programme fut jugé irréaliste et pléthorique. Comme en 1983, les Britanniques risquent de ne retenir que les promesses les plus symboliques telles que la gratuité de la fibre optique, le passage à la semaine de 4 jours ou l'abolition de la Chambre des Lords alors que le parti proposait un programme original de transformation sociale et une révolution industrielle verte. S'il veut espérer un jour reprendre les rênes du pouvoir, le parti travailliste devra se montrer plus réaliste et reconstruire sa réputation de compétence en économie, largement ternie depuis la crise de 2008.

Toufik Abdou est doctorant en civilisation britannique à l'Université Sorbonne Nouvelle Paris III, Paris. 


\section{BIBLIOGRAPHIE}

ALEXANDRE-COLLIER Agnès, AVRIL Emmanuelle, Les Partis Politiques en Grande-Bretagne, Paris : Armand Colin, 2013.

BBC News, 11 avril 2018, «Jeremy Corbyn pledges free bus travel for under-25s ».

BOLTON Matt \& PITTS Frederick Harry, Corbynism: A Critical Approach, Bingley: Emerald Publishing, 2018.

BOND Becky, Rules for Revolutionaries: How Big Organizing Can Change Everything, White River Junction: Chelsea Green Publishing, 2016.

FIELDING Steven, The Labour Party: Continuity and Change in the Making of 'New Labour' Londres: Palgrave, 2002.

FINER Samuel Edward,1975, Adversary politics and electoral reform, Londres: Anthony Wigram, 1975. GMB, 18 janvier 2017, «Justice for Cammell Lairds 37 ».

HART Keith, « Karl Polanyi : prophète de la fin de l'économie libérale », Revue Interventions économiques [Online], 38 | 2008, URL: http://journals.openedition.org/interventionseconomiques/ 304 ; DOI : https://doi.org/10.4000/interventionseconomiques.304.

IFS, Institute for Fiscal Studies, « Labour manifesto: an initial reaction from IFS researchers ».

ITV News, 16 mai 2017, « Jeremy Corbyn launches Labour's election manifesto ».

King's College London, 20 février 2020, « 120 years of the Labour Party: In conversation with Tony Blair

Klug Adam, Rees Emma, Schneider James, « Momentum: a new kind of politics », Renewal, Vol.24, number 2, 2016.

LACLAU Ernesto, On Populist Reason, London: Verso, 2005.

Le Vent Se Lève, Lenny Benbera, 2 octobre 2019, « Laura Parker : Momentum est une organisation d'origine populaire».

Mediapart, Fabien Escolana, « Défaite de Corbyn : la mission était impossible », 19 décembre 2019.

MOUFFE Chantal, Agnostics: Thinking the World Politically, London, Verso, 2013.

LAPAVITSAS Costas, The Left Case against the EU, Londres, Polity Press, 2018.

Le Temps, Richard Baldwin, 21 décembre 2016, « Inévitablement, la mondialisation va s'accélérer ».

MANIFESTO PROJECT, Project description, URL= https://manifesto-project.wzb.eu.

McDONALD Andy, 13 février 2020, « Telling the Truth About Buses », Tribune mag.

MSNBC, « Naomi Klein On a 'Yes' Agenda To Counter President Donald Trump », 12 juin 2017.

Open Democracy, 16 novembre 2019, « Broadband communism'? Outside the UK, public broadband is a raving success ».

POLANYI Karl, La Grande Transformation, Gallimard : Paris, 1983.

PRINCE Rosa, Comrade Corbyn, Londres, Biteback Publishing, 2015

Regards.fr, Laura Raim, 23 septembre 2016, « Corbyn, l'ancien et les modernes ». 
SRNICEK Nick \& WILLIAMS Alex, Inventing the Future: Postcapitalism and a World Without Work, Londres: Verso, 2016.

The Economist; 19 octobre 2017. «Preston, Jeremy Corbyn's model town ».

The Guardian, 22 juillet 2015, « Tony Blair: If your heart's with Jeremy Corbyn, get a transplant ».

The Guardian, 7 décembre 2015, « The dirty tricks of the Shrewsbury trials expose the dark heart of the radical 1970s».

The Guardian, 18 mai 2017, « The scandal of Orgreave ».

The Guardian, 9 juin 2017, « Was it the Corbyn memes wot won it? Here are some of the best? ».

The Guardian, 10 juin 2017, « How Jeremy Corbyn turned a youth surge into general election votes ».

The Guardian, Jeremy Corbyn, 14 décembre 2019, «We won the argument, but I regret we didn't convert that into a majority for change ".

The Independent, 8 février 2020, «Corbyn's 'cabal' of top aides sabotaged election campaign, says

Labour leader's close friend ».

The Labour Party, Alternative Models of Ownership.

The Labour Party, 25 avril 2019, «Labour announces new funding to reverse Tory cuts to 3,000 bus routes».

The Labour Party, 15 novembre 2019, «Jeremy Corbyn's Speech on the Labour's British Broadband announcement ».

The Labour Party Manifesto 2010, «A future fair for all ».

The Labour Party Manifesto 2019, «It's Time For Real Change ».

The University of Sheffield, «UK has higher level of regional inequality than any other large wealthy country».

The World Transformed, Programme 2019, https://theworldtransformed.org/festival/2019/ programme.

WALUMBW F.O., AVOILOI B.J., GARDNER W.L., WERSINGE T.S. \& PETERSON S.J., « Authentic leadership: Development and validation of a theory-based measure ", Journal of Management, 34 (1), 2008.

\section{NOTES}

1. The Observer, Jeremy Corbyn, "We won the argument, but I regret we didn't convert that into a majority for change”, 14 décembre 2019, https://www.theguardian.com/politics/2019/dec/14/wewon-the-argument-but-i-regret-we-didnt-convert-that-into-a-majority-for-change, , [consulté le 2 mai 2020].

2. Ibid.

3. Alexandre-Collier Agnès, Avril Emmanuelle, Les Partis Politiques en Grande-Bretagne, Paris: Armand Colin, 2013, p. 78.

4. Ibid.

5. Ibid.

6. Finer Samuel Edward,1975, Adversary politics and electoral reform, Londres: Anthony Wigram, 1975, p. 270-274. 
7. Manifesto Project, Project description, URL= https://manifesto-project.wzb.eu, [consulté le 04/05/2020].

8. citation attribuée au député travailliste Gerald Kaufman en 1983.

9. Données compilées par le MARPOR.

10. The University of Sheffield, UK has higher level of regional inequality than any other large wealthy country, https://www.sheffield.ac.uk/news/nr/uk-higher-regional-inequality-large-wealthycountry-1.862262, , [consulté le 1 mai 2020].

11. Polanyi Karl, La Grande Transformation, Gallimard : Paris, 1983.

12. Hart Keith, «Karl Polanyi : prophète de la fin de l'économie libérale », Revue Interventions économiques [Online], $\quad 38 \mid 2008, \quad$ URL: http://journals.openedition.org/ interventionseconomiques/304; DOI : https://doi.org/10.4000/interventionseconomiques.304, [consulté le 2 mai 2020].

13. Lapavitsas Costas, The Left Case against the EU, Londres, Polity Press, 2018.

14. Bolton Matt \& Harry Pitts Frederick, Corbynism: A Critical Approach, Bingley, Emerald Publishing p.5.

15. The Labour Party Manifesto 2019, p. 19.

16. BBC News, 11 avril 2018, Jeremy Corbyn pledges free bus travel for under-25s, https:// www.bbc.co.uk/news/uk-politics-43726983, [consulté le 2 mai 2020].

17. McDonald Andy, Telling the Truth About Buses, Tribune mag, https://tribunemag.co.uk/ 2020/02/telling-the-truth-about-buses, [consulté le 2 mai 2020].

18. The Labour Party, Labour announces new funding to reverse Tory cuts to 3,000 bus routes, 25 avril 2019, https://labour.org.uk/press/labour-announces-new-funding-reverse-tory-cuts-3000-busroutes/, , [consulté le 6 mai 2020].

19. The Economist; 19 octobre 2017. Preston, Jeremy Corbyn's model town, https:// www.economist.com/britain/2017/10/19/preston-jeremy-corbyns-model-town, [consulté le 6 mai 2020].

20. The Financial Times, Labour to water down some radical policies for election manifesto, 11 novembre 2019, https://www.ft.com/content/00c2a854-0180-11ea-b7bc-f3fa4e77dd47, [consulté le 6 mai 2020].

21. Fielding Steven, The Labour Party: Continuity and Change in the Making of 'New Labour' Londres, Palgrave, 2002, p.35.

22. Institute for Fiscal Studies, Labour manifesto : an initial reaction from IFS researchers https:// www.ifs.org.uk/election/2019/article/labour-manifesto-an-initial-reaction-from-ifs-researchers, [consulté le 6 mai 2020].

23. Prince Rosa, Comrade Corbyn, Londres, Biteback Publishing, 2015, p. 268.

24. Document distribué lors d'une réunion qui s'est tenue pendant le Congrès travailliste de 2019 à Brighton.

25. The Labour Party, Alternative Models of Ownership, p. 5.

26. Ibid.

27. The Labour Party, Alternative Models of Ownership, p. 9.

28. Bolton Matt \& Harry Pitts Frederick, Corbynism: A Critical Approach, Bingley, Emerald Publishing, p.5.

29. Srnicek Nick \& Williams Alex, Inventing the Future: Postcapitalism and a World Without Work, Londres, Verso, 2016.

30. Le Temps, Richard Baldwin: "Inévitablement, la mondialisation va s'accélérer", 21 décembre 2016, $\mathrm{URL}=$ https://www.letemps.ch/economie/richard-baldwin-inevitablement-mondialisation-vasaccelerer, [consulté le 5 mai 2020].

31. The Labour manifesto 2019, p. 6. 
32. The Guardian, "How Jeremy Corbyn turned a youth surge into general election votes", 10 juin 2017, URL=https://www.theguardian.com/politics/2017/jun/10/jeremy-corbyn-youth-surge-votesdigital-activists, [consulté le 12 mai 2020].

33. Buzzfeed, "Here's How Labour Ran An Under-The-Radar Dark Ads Campaign During The General Election", 6 juillet 2007, URL= https://www.buzzfeed.com/jimwaterson/heres-howlabour-ran-an-under-the-radar-dark-ads-campaign?utm_term=.qxrBO0aNEp\#.juo20pAqne, [consulté le 12 mai 2020].

34. The Guardian, “Was it the Corbyn memes wot won it? Here are some of the best?", 9 juin 2017, https://www.theguardian.com/politics/2017/jun/09/corbyn-memes-wot-won-it-some-of-thebest, [consulté le 8 mai 2020]

35. Open Democracy, 'Broadband communism'? Outside the UK, public broadband is a raving success", 16 novembre 2019, https://www.opendemocracy.net/en/oureconomy/broadband-communismoutside-the-uk-public-broadband-is-a-raving-success/, [consulté le 8 mai 2020].

36. Labour Party, Jeremy Corbyn's Speech on the Labour's British Broadband announcement; 15 novembre 2019, https://labour.org.uk/press/full-text-of-jeremy-corbyns-speech-on-laboursbritish-broadband-announcement/, [consulté le 8 mai 2020].

37. Regards.fr, Laura Raim, Corbyn, l'ancien et les modernes, 23 septembre 2016, URL=http:// www.regards.fr/archives/web/article/corbyn-l-ancien-et-les-modernes, [consulté le 8 mai 2020]. 38. The Labour Party Manifesto 2019, p. 99: "We believe effective diplomacy can address the drivers of conflict and insecurity."

39. The Labour Manifesto 2019, p.38: "We will reverse cuts to Sure Start and create a new service, Sure Start Plus, with enough centres to provide a genuinely universal service, available in all communities, focused on the under-2s."

40. ITV News, “Jeremy Corbyn launches Labour's election manifesto”, 16 mai 2017, URL= https:// www.youtube.com/watch?v=8Rzz81Rebeg, [consulté le 6 mai 2020].

41. The Labour party Manifesto 2019, p.72.

42. Ibid.

43. Ibid.

44. The Labour Party Manifesto 2019, p.11.

45. The Labour Party Manifesto 2019., p.74.

46. The Labour Party Manifesto 2010, p. 4:5: "We will establish a new National Care Service working in partnership with the NHS to transform the way care is provided to the elderly and disabled people."

47. ITV News, 16 mai 2017, Jeremy Corbyn launches Labour's election manifesto, URL= https:// www.youtube.com/watch?v=8Rzz81Rebeg, [consulté le 10 mai 2020].

48. Walumbw F.O., Avoilio B.J., Gardner W.L., Wersing T.S. \& Peterson S.J., Authentic leadership: Development and validation of a theory-based measure, Journal of Management, 34 (1), 2008, p. 94.

49. Prince Rosa, Op. cit. p. 50.

50. The Guardian, Tony Blair : If your heart's with Jeremy Corbyn, get a transplant, 22 juillet 2015, URL= https://www.theguardian.com/politics/video/2015/jul/22/tony-blair-jeremy-corbyn-labour-

leadership-video, [consulté le 11 mai 2020].

51. The Labour Party Manifesto 2019, p.73.

52. The Labour Party Manifesto 2019, p.81.

53. Laclau Ernesto, 2005, On Populist Reason, London, Verso.

54. Mouffe Chantal, 2013, Agnostics: Thinking the World Politically, London, Verso.

55. The Labour Party Manifesto 2019, p.61.

56. The Guardian, The scandal of Orgreave, URL= https://www.theguardian.com/politics/2017/ may/18/scandal-of-orgreave-miners-strike-hillsborough-theresa-may, [consulté le 10 mai 2020]. 57. The Guardian, The dirty tricks of the Shrewsbury trials expose the dark heart of the radical 1970s, $\mathrm{URL}=\quad$ https://www.theguardian.com/commentisfree/2015/dec/07/shrewsbury-trials-1970strade-unionists-ricky-tomlinson-edward-heath, [consulté le 10 mai 2020]. 
58. GMB, Justice for Cammell Lairds 37, URL= http://www.gmbnorthwest.co.uk/news/justicecammell-lairds-37, [consulté le 10 mai 2020].

59. The Labour Party Manifesto 2019, p. 60.

60. Prince Rosa, Op. cit., p.299.

61. King's College London, 120 years of the Labour Party: In conversation with Tony Blair, https:// www.youtube.com/watch?v=xle3C36bAvI, [consulté le 3 mai 2020].

62. The Independent, Corbyn's 'cabal' of top aides sabotaged election campaign, says Labour leader's close friend, 8 février 2020, URL=https://www.independent.co.uk/news/uk/politics/corbyngeneral-election-labour-alan-simpson-seamus-milne-karie-murphy-a9324066.html, [consulté le 10 mai 2020].

63. Le Vent Se Lève, Lenny Benbera, Laura Parker: Momentum est une organisation d'origine populaire, URL=https://lvsl.fr/laura-parker-momentum-est-une-organisation-dorigine-populaire, [consulté le 10 mai 2020].

64. The World Transformed, Programme https://theworldtransformed.org/festival/2019/ programme, [consulté le 10 mai 2020].

65. Klug Adam, Rees Emma, Schneider James, Momentum: a new kind of politics, Renewal, Vol.24, number 2, 2016.

66. Bond Becky, Rules for Revolutionaries: How Big Organizing Can Change Everything, White River Junction, Chelsea Green Publishing, 2016.

67. MSNBC, Naomi Klein On a 'Yes' Agenda To Counter President Donald Trump, 12 juin 2017 https:// www.youtube.com/watch?v=BxrYBVsK2vc, [consulté le 3 mai 2020].

68. The Labour Party Manifesto 2019, p.92.

69. BBC Politics Live, 16 décembre 2019.

70. Mediapart, Fabien Escolana, Défaite de Corbyn : la mission était impossible, 19 décembre 2019, URL=https://www-mediapart-fr.acces-distant.sciencespo.fr/journal/international/191219/ defaite-de-corbyn-la-mission-etait-impossible?page_article=2, [consulté le 3 mai 2020]. 71. YouGov, Matthew Smith, Labour economic policies are popular, so why aren't Labour, URL=https:// yougov.co.uk/topics/politics/articles-reports/2019/11/12/labour-economic-policies-arepopular-so-why-arent-, [consulté le 3 mai 2020].

\section{RÉSUMÉS}

Le glossaire du Parlement britannique définit le programme comme « un document publié par un parti politique avant une élection législative ", ajoutant qu'il " contient un ensemble de mesures que le parti [...] souhaiterait appliquer une fois élu». Les programmes sont un rituel de la vie politique britannique. Publié trois semaines avant la date de l'élection législative, le " manifesto » est le résultat de tractations, compromis et négociations pendant de long mois voire plusieurs années au sein du parti travailliste. Le programme électoral est souvent un enjeu politique dans la mesure où il cristallise les divisions internes au sein du parti travailliste, les différents courants du parti souhaitant imprimer leur marque.

«Le temps est venu pour un vrai changement ». C'est le titre donné par le chef du parti travailliste, Jeremy Corbyn, à son programme pour les élections législatives du 12 décembre 2019. Parmi les mesures annoncées, les nationalisations du rail, de l'eau et de la poste tout comme d'une partie du géant des télécommunications BT afin de mettre en œuvre l'une des promesses les plus 
spectaculaires du parti : fournir le haut débit gratuit pour tous. Cette dernière mesure a été très vite raillée par la presse et le parti conservateur et utilisée par la frange modérée du parti travailliste pour dénoncer le manque de réalisme de l'équipe Corbyn.

Cet article tentera d'analyser les propositions du programme travailliste 2019 en mettant en lumière les fondements théoriques qui les sous-tendent. Une comparaison avec le programme de 2017 permettra de comprendre les raisons de l'échec de 2019 et de définir les contours du Corbynisme.

The glossary which can be found on the UK Parliament website defines the manifesto as "a publication issued by a political party before a general election", adding that "it contains the set of policies that the party stands for and would wish to implement if elected to govern". Election manifestos are part of the ritual of British general elections: they provide a compendium of valid party positions and are used as campaign material. Published three weeks before the date of the general election, the manifesto is the result of agreements and negotiations that take place over the course of several months or even several years within the Labour party. Writing the manifesto is also an exercise in party management, reflecting the balance of power between the different factions of the party.

"It's Time For Real Change". This is the title given by Labour leader Jeremy Corbyn to his manifesto for the general election that took place on 12 December 2019. The document included plans to take parts of Britain's energy industry back into public ownership alongside the railways and the Royal Mail as well as the pledge to nationalize part of BT and provide free broadband across the UK. This policy was vilified by the media, mocked by the soft left and attacked by Boris Johnson as a "crazed communist scheme".

This paper will analyse the key policies of the 2019 Labour manifesto and highlight their theoretical framework. A comparison with the 2017 manifesto will enable us to define the contours of what Corbynism was and help us understand the reasons for the defeat.

\section{INDEX}

Mots-clés : parti travailliste, élections législatives, Jeremy Corbyn

Keywords : Labour Party, manifesto, general elections, Jeremy Corbyn

\section{AUTEUR}

\section{TOUFIK ABDOU}

Université Sorbonne Nouvelle Paris III CREW EA 4399 